CHAPITRE 9

\title{
LA CONCEPTION DES DISPOSITIFS DE FRANCHISSEMENT POUR LES ALOSES
}

\author{
M. LARINIER* et F. TRAVADE**
}

\footnotetext{
* CSP-CEMAGREF, GHAAPPE - Institut de Mécanique des Fluides - Avenue du Professeur Camille Soula - 31400 TOULOUSE

** EDF - Etudes et Recherches - 6, Quai Watier - 78401 CHATOU CEDEX
}

\section{INTRODUCTION}

En France, jusqu'à ces dernières années, la plupart des passes à poissons se sont révélées inefficaces pour l'alose (alosa alosa) et l'installation de barrages sur les cours d'eau à aloses s'est pratiquement toujours traduite par la raréfaction, voire la disparition, de cette espèce en amont. Ce phénomène n'est pas spécifique à la France : en 1923, un commissaire du Bureau des Pêcheries aux Etats-Unis écrivait au sujet de l'alose américaine (alosa sapidissima) "qu'il était douteux qu'une alose remonte un jour une échelle à poissons quelle qu'elle soit" et jusqu'en 1955, sur le continent nord-américain, les seuls dispositifs jugés efficaces pour cette espèce étaient les deux passes gigantesques situées au barrage de Bonneville sur la rivière Columbia, passes d'une dizaine de mètres de largeur ayant coûté près de $10 \%$ du coût de l'aménagement hydroélectrique (DALLEY, 1980).

Il a semblé intéressant d'intégrer dans la suite l'expérience acquise pour les deux espèces (alosa alosa et alosa sapidissima), dans la mesure où toutes les observations effectuées, soit en France, soit en Amérique du Nord, indiquent que leur comportement migratoire est très voisin.

\section{CAPACITÉ DE NAGE ET COMPORTEMENT MIGRATOIRE DE L'ALOSE}

\subsection{Capacité de nage}

L'alose est moins bonne nageuse que le saumon ou la truite de mer: les observations (C.T.G.R.E.F., 1981) montrent que des vitesses de l'ordre de $2 \mathrm{~m} / \mathrm{s}$ constituent une difficulté majeure pour un certain nombre d'entre elles dès lors que la distance à franchir dépasse quelques dizaines de mètres.

Les vitesses de sprint ont pu être estimées lors d'observations sur le seuil de StLaurent-des-Eaux sur la Loire (LITAUDON, 1985) : à la température de $16^{\circ} \mathrm{C}-17^{\circ} \mathrm{C}$, des vitesses variant de $3.1 \mathrm{~m} / \mathrm{s}$ à $4.7 \mathrm{~m} / \mathrm{s}$ suivant les individus pouvaient être soutenues pendant une durée voisine de 6.5 secondes. Les vitesses maximales de nage que l'alose ne pouvait soutenir que quelques secondes ont été estimées à des valeurs comprises entre $4.1 \mathrm{~m} / \mathrm{s}$ et $6.1 \mathrm{~m} / \mathrm{s}$.

Ces chiffres sont à rapprocher de ceux concernant l'alose américaine. Des observations (WEAVER, 1965) ont été effectuées sur les distances nagées par plus de 800 individus dans un canal d'une trentaine de mètres de longueur, dans lequel les vitesses d'écoulement variaient de $3.5 \mathrm{~m} / \mathrm{s}$ à $4.15 \mathrm{~m} / \mathrm{s}$. Aucune alose n'a pu franchir ce canal dans sa totalité. Les distances moyennes parcourues, lorsque la vitesse moyenne dans le canal était de $3.5 \mathrm{~m} / \mathrm{s}, 3.85 \mathrm{~m} / \mathrm{s}$ et $4 \mathrm{~m} / \mathrm{s}$, étaient respectivement de $9 \mathrm{~m}, 7.1 \mathrm{~m}$ et $5.7 \mathrm{~m}$, et cela dans des conditions thermiques a priori favorables $\left(21^{\circ} \mathrm{C}\right)$. A titre de comparaison, toutes les truites steelhead, dont les performances de nage sont comparables à celles du saumon atlantique, ont franchi sans problème toute la longueur du canal. D'après ces données, les vitesses maximales de nage pour la majorité des individus varieraient entre $4.3 \mathrm{~m} / \mathrm{s}$ à 
$4.6 \mathrm{~m} / \mathrm{s}, 10 \%$ des individus ayant des vitesses de sprint inférieures à $3.9 \mathrm{~m} / \mathrm{s}$ ou supérieures à $4.9 \mathrm{~m} / \mathrm{s}$.

Si l'on adapte à l'alose des modèles semi-empiriques proposés par BEACH (1984), on obtient, en paramétrant suivant la température, un faisceau de courbes qui concorde avec les observations effectuées sur le terrain. Les vitesses maximales de nage à $20^{\circ} \mathrm{C}$ varieraient de $4.0 \mathrm{~m} / \mathrm{s}$ à $5.4 \mathrm{~m} / \mathrm{s}$ suivant la taille des individus, l'endurance correspondante variant de 5 à 16 secondes. Pour des températures plus basses, les valeurs de la vitesse maximale de nage sont notablement réduites, se situant à $15^{\circ} \mathrm{C}$ entre $3.5 \mathrm{~m} / \mathrm{s}$ et $4.3 \mathrm{~m} / \mathrm{s}$. La limite supérieure de la vitesse de croisière varierait suivant la taille de $0.80 \mathrm{~m} / \mathrm{s}$ à $1.4 \mathrm{~m} / \mathrm{s}$.

Des vitesses de l'ordre de $3.5 \mathrm{~m} / \mathrm{s}$ à $4 \mathrm{~m} / \mathrm{s}$ sur quelques mètres constituent donc une barrière difficilement franchissable pour l'alose : or il suffit d'une dénivellation de l'ordre du mètre entre les niveaux amont et aval de part et d'autre d'un obstacle pour que ces vitesses soient atteintes : cela signifie que des seuils de hauteurs modestes peuvent constituer des obstacles sérieux à la migration de l'alose, surtout si l'écoulement se fait à "jet plongeant" comme on le verra par la suite.

\subsection{Comportement migratoire}

L'alose est un poisson de surface : il convient donc de lui offrir dans les dispositifs de franchissement un passage en surface.

Elle se déplace en bancs : il faut lui offrir les passages les plus larges possibles pour éviter de déstructurer ces bancs.

Le piégeage d'aloses dans un volume d'eau trop restreint, même de courte durée, peut entraîner des mortalités importantes.

L'alose semble rechercher les veines d'eau régulières à filets parallèles, même à vitesse importante. Elle semble éviter dans la mesure du possible les zones tourbillonnaires à forte turbulence et les eaux émulsionnées (remous à l'aval d'une chute, ressaut, aval des turbines) dans lesquelles elle évolue avec beaucoup plus de difficulté que les salmonidés.

L'alose ne saute pas comme le font la plupart des salmonidés et ne franchit généralement les obstacles qu'en nageant. C'est pourquoi elle éprouve de très grosses difficultés à franchir les écoulements à "jet plongeant", dans lesquels elle a tendance a être désorientée, se positionnant tête vers l'aval dans le contre-courant existant en surface.

Un certain nombre d'observations montrent que l'alose demeure facilement piégée dans les encoignures et dans les zones de recirculation engendrées par les décrochements des murs des ouvrages de franchissement ; ces observations ont aussi bien été effectuées sur l'alose américaine aux Etats-Unis que sur la grande alose en France (C.T.G.R.E.F., 1981). En conséquence, il est important, dans la mesure du possible évidemment, de lui assurer un passage le long des parois en minimisant le volume des zones de recirculation, tout en évitant évidemment les phénomènes de court-circuit qui seraient préjudiciables au fonctionnement hydraulique de la passe.

Le comportement de l'alose dans les passes à poissons est caractérisé par des allers et retours fréquents ("fall-back activity") pouvant se traduire par la dévalaison d'un banc entier lorsque les poissons se trouvent piégés par des écoulements particuliers (zone de recirculation à axe vertical créée par un décrochement latéral ou un changement de direction de la passe ou à axe horizontal créé par un orifice noyé).

L'observation visuelle sur plusieurs sites ainsi que différentes études de radiopistage ont permis de mettre en évidence le fait que l'alose prospectait beaucoup moins activement que les salmonidés au pied d'un obstacle et qu'elle était susceptible de rester bloquée très longtemps dans une zone "attractive".

L'alose semble d'autre part très sensible aux variations brutales de luminosité : il paraît indispensable d'éclairer les tronçons souterrains et plus généralement les zones particulièrement sombres des dispositifs de franchissement. 


\section{LES PASSES A BASSINS}

En prenant un certain nombre de précautions particulières, les passes à bassins successifs peuvent s'avérer efficaces pour l'alose.

L'écoulement doit être à "jet de surface", et non à "jet plongeant", le poisson s'orientant à partir de la configuration des courants de surface.

Les orifices noyés sont à proscrire, l'alose tendant à rester piégée dans les contrecourants situés en surface au-dessus des orifices : certaines sections de régulation par orifice noyé ont du être abandonnées sur la côte Ouest des USA suite aux blocages et mortalités observées (MONK et al., 1989). Des blocages d'aloses ont également été observés dans la section de régulation amont de la passe à ralentisseurs du Bazacle (DARTIGUELONGUE, 1990).

Le passage des poissons doit être assuré le long des parois, les échancrures centrales étant à éviter : il convient de prévoir au moins une fente ou échancrure latérale, le mieux étant d'assurer le passage de chaque côté du dispositif.

Il faut éviter, dans la mesure du possible, la présence de zones de recirculation importantes dans lesquelles le poisson aurait tendance à rester piégé (passes de Rainbow sur la rivière Farmington, Turners Falls sur la rivière Connecticut, Tuilières sur la Dordogne), en particulier dans les bassins où s'effectue un changement de direction de la passe, bassins qui sont généralement plus volumineux et d'une hydrodynamique bien particulière. Dans la mesure où il est difficile d'éliminer ces zones, il est possible d'en interdire l'accès au poisson à l'aide de grilles (DARTIGUELONGUE et al., 1992).

Les poissons se déplacent en bancs et il faut faire en sorte que la passe ne déstructure pas trop ces bancs ; il convient d'adopter des bassins de volume important et des passages les plus larges possibles.

Les échancrures ou les fentes doivent avoir une largeur minimale de $0.45 \mathrm{~m}$ à $0.50 \mathrm{~m}$.

Les bassins doivent être suffisamment profonds ( $1.20 \mathrm{~m}$ au minimum) et peu turbulents : une puissance dissipée volumique de 150 watts $/ \mathrm{m}^{3}$ paraît être un maximum.

Les chutes ne doivent pas dépasser $0.30 \mathrm{~m}$. II est préférable d'utiliser des chutes inférieures (de $0.20 \mathrm{~m}$ à $0.25 \mathrm{~m}$ ), chutes pour lesquelles il sera plus facile de créer des écoulements à "jet de surface" tout en conservant des passages relativement larges.

Si l'on cherche à respecter ces différentes contraintes, on aboutit généralement à des ouvrages larges, transitant des débits importants (supérieurs à $1 \mathrm{~m}^{3} / \mathrm{s}$ ), le volume des bassins dépassant généralement une douzaine de $\mathrm{m}^{3}$. (Fig. 1):

On citera quelques exemples d'ouvrages qui se sont révélés efficaces pour l'alose

- passes de type Ice Harbor sur les rivières Columbia (Bonneville, John Day...) et Connecticut (Turners Falls) aux USA, à condition de porter la charge sur les seuils à une quarantaine de $\mathrm{cm}$ de façon à tendre vers un écoulement de surface (RIDEOUT et al., 1985). L'augmentation de la charge de $0.30 \mathrm{~m}$ à $0.40 \mathrm{~m}$, dans le cas de bassins relativement courts, oblige à obturer une échancrure sur deux, en quinconce, de façon à éviter les phénomènes de court-circuit et diminuer la turbulence dans les bassins,

\section{Dordogne),}

— passes à deux fentes verticales de $0.55 \mathrm{~m}$ de largeur (passe de Bergerac sur la

- passes à une fente verticale ou à échancrure latérale profonde, à condition d'adopter des largeurs de fentes suffisantes, de l'ordre de $0.45 \mathrm{~m}$ à $0.50 \mathrm{~m}$ (passes du Ramier sur la Garonne et de Sainte-Livrade sur le Tarn). Dans ce type de passe à fente verticale, il faut veiller à minimiser l'étendue des zones de recirculation en réduisant au maximum le rapport largeur/longueur des bassins et en veillant à orienter convenablement les jets dans les bassins vers le tiers aval de la paroi latérale. On cherchera à réduire autant que possible la hauteur des seuils dans les fentes.

- passes à "chicanes", du type utilisé dans les sections de régulation de certains gros ouvrages : passe de Vernon sur la rivière Connecticut, Bonneville et John Day sur la 
rivière Columbia (MONK et al., 1989). Le gros avantage de ce type de passes est la quasiabsence de zones de recirculation. Cela se traduit cependant par un ouvrage très volumineux, compte tenu de la faible chute acceptable (maximum d'une quinzaine de $\mathrm{cm}$ ) entre chicanes successives.

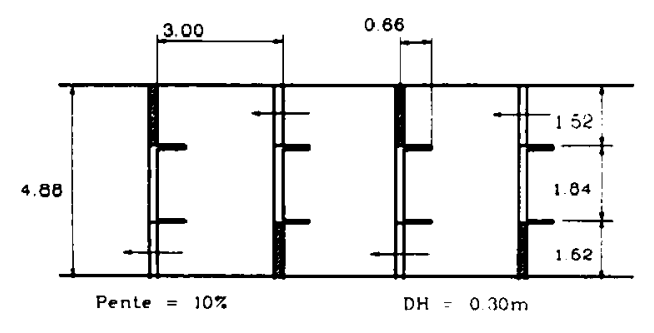

PASSE DE TURNERS FALLS - (riviere Connecticut)
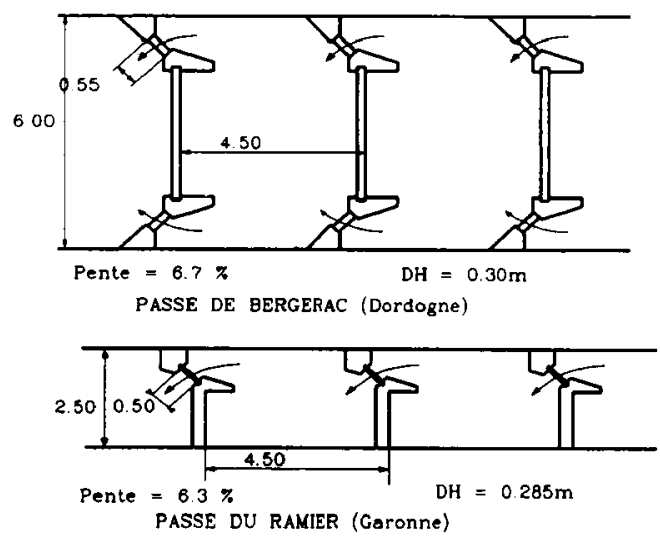

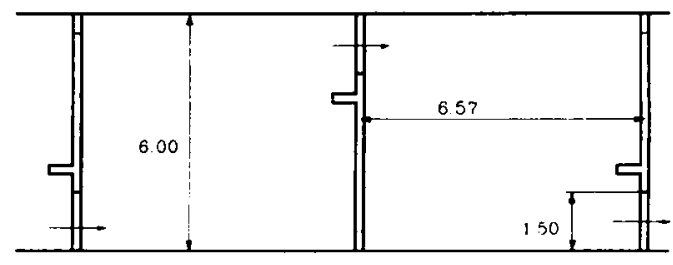

$\mathrm{DH}=0.30 \mathrm{~m}$

PASSE DE SAINTE-LIVRADE (Tarn)

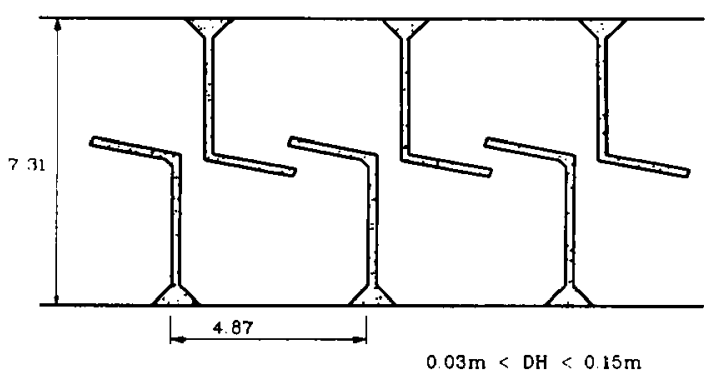

SECTION DE CONTROLE AMONT AU BARRAGE DE JOHN DAY (Columbia)

Figure 1 : Passes à bassins empruntées par l'alose.

Figure 1 : Pool fishways utilized by shad.

\section{LES PASSES A RALENTISSEURS}

Bien qu'il existe, aussi bien en France qu'aux USA ou au Canada, plusieurs passes à ralentisseurs empruntées par l'alose, il est cependant difficile de se prononcer de façon très tranchée sur l'efficacité de tels dispositifs pour cette espèce.

L'observation montre de façon évidente que l'alose négocie beaucoup moins bien que les salmonidés les écoulements "en hélice" caractérisant les passes à ralentisseurs.

Plusieurs centaines d'aloses ont été contrôlées dans une passe à ralentisseurs plans (pente $13 \%$, largeur $1.22 \mathrm{~m}$, longueur $12 \mathrm{~m}$ ) sur la rivière Narraguagus (DALLEY, 1980).

La passe à ralentisseurs plans de Beaucaire (deux volées de pente $8 \%$ et $10 \%$, largeur $1.30 \mathrm{~m}$, longueur totale $40 \mathrm{~m}$ ) sur le Rhône s'est révèlée très peu efficace pour l'alose (C.T.G.R.E.F.,1981). Le fait que cet ouvrage ne fonctionne pas ne peut être cependant attribué aux seules conditions hydrauliques dans la passe. On pourrait aussi expliquer en grande partie cet échec par les conditions de piègeage difficiles, par le passage en galerie et l'obscurité de la sortie de la passe, et surtout par l'attractivité problématique du dispositif (entrée située à $1500 \mathrm{~m}$ du point de blocage des migrateurs, débit complémentaire d'attrait très turbulent ne guidant pas le poisson vers l'entrée de la passe). 
Chaque année plusieurs milliers d'aloses se présentent au pied du barrage du Bazacle à Toulouse, équipé d'une passe à bassins successifs à fentes verticales et d'une passe à ralentisseurs à chevrons épais comportant 3 volées d'une douzaine de mètres de longueur (pente $17 \%$, largeur 1.50 m) (DARTIGUELONGUE, 1991, 1992). Les aloses empruntant la passe à ralentisseurs ne constituent cependant qu'un faible pourcentage (de l'ordre de $1 \%$ ) des aloses franchissant le barrage, la grande majorité empruntant la passe à bassins successifs. La meilleure situation de l'entrée de la passe à bassins entre cependant pour une part sans doute significative dans la différence d'efficacité des deux dispositifs.

Sur un contre-canal du Rhône, une passe à ralentisseurs à chevrons épais (pente $16 \%$, largeur $1.80 \mathrm{~m}$, longueur $10 \mathrm{~m}$, section de chevrons $0.10 \mathrm{~m} \times 0.10 \mathrm{~m}$ ) a été testée à titre expérimental (BARIL, 1988). Plus de 350 aloses l'ont franchie, pour des débits unitaires transitant dans la passe variant de $300 \mathrm{l} / \mathrm{s} / \mathrm{m}$ à $750 \mathrm{l} / \mathrm{s} / \mathrm{m}$. L'efficacité était cependant très liée aux conditions à l'entrée de la passe : ce n'est que lorsque les aloses étaient guidées vers cette passe par un filet qu'elles l'empruntaient. En l'absence de filet, les observations ont montré que les aloses préféraient se tenir à l'écart des zones turbulentes situées à l'entrée de la passe.

Plusieurs tests ont été effectués pour comparer les efficacités respectives sur un même site de passes à ralentisseurs et de passes à bassins.

Au Canada (CONRAD, com. pers.), des expérimentations ont montré que des aloses utilisaient de préférence une passe à ralentisseurs plans plutôt qu'une passe à bassins installée à proximité ; ce résultat peut être attribué d'une part à la plus grande attractivité de la passe à ralentisseurs (débit beaucoup plus important), d'autre part à l'exiguitté de la passe à bassins, encore moins appropriée à l'alose que la passe à ralentisseurs.

Des essais ont été effectués au laboratoire de Bonneville pour étudier la possibilité d'utiliser des passes à ralentisseurs afin d'orienter des migrateurs d'une passe à bassins vers un dispositif de détection de marques magnétiques (SLATICK, 1975). La passe testée est une passe à ralentisseurs type Alaska (pente $24 \%$, largeur $0.56 \mathrm{~m}$, largeur libre $0.35 \mathrm{~m}$, longueur $8 \mathrm{~m}$ ) débitant au maximum $160 \mathrm{l} / \mathrm{s}$ à plein bord. Ces expérimentations ont montré que les aloses éprouvaient de façon générale beaucoup plus de répulsion que les salmonidés à s'engager dans l'ouvrage. Une forte proportion d'aloses franchissaient cependant la passe à la condition qu'un temps suffisant et que des conditions hydrauliques appropriées leur soient donnés en entrée et en sortie de la passe. En particulier, les résultats les plus significatifs ont été obtenus lorsque le poisson était guidé vers l'entrée de la passe par une grille inclinée. Lorsque le choix leur était offert entre une passe à bassins (à échancrure centrale et à "jet plongeant") et la passe à ralentisseurs, toutes les aloses et la majorité des salmonidés empruntaient cette dernière.

Ces résultats sur les efficacités comparatives des passes à ralentisseurs et des passes à bassins - toujours au profit des premières - sont à pendre avec beaucoup de prudence, car il s'agissait de passes à bassins de petites dimensions et surtout de passes à "jet plongeant" a priori peu favorables au passage des aloses : les résultats auraient sans doute été tout autres si les passes à bassins avaient été de plus grandes dimensions, à débit comparable à celui de la passe à ralentisseurs et surtout à écoulement à "jet de surface".

\section{LES ASCENSEURS ET ECLUSES}

Le piégeage d'aloses dans un volume d'eau trop restreint, même de courte durée, peut entraîner des mortalités importantes. C'est pourquoi, dans les ascenseurs (TRAVADE et al., 1992), on piège l'alose dans un bassin de stabulation de dimensions suffisantes (dimensions mimimales de l'ordre de $5 \mathrm{~m} \times 2.5 \mathrm{~m} \times 1.5 \mathrm{~m}$ ) en prévoyant un volume d'eau mimimum (environ 30 l/alose) pour chaque individu présent. II est difficile d'envisager, comme pour les salmonidés, un piégeage dans une nasse sauf si celle-ci est de taille suffisante, du même ordre de grandeur que le bassin de stabulation.

On est donc amené à prévoir un système de concentration (grille montée sur un chariot mobile) amenant lors de l'opération de transfert le poisson au-desssus de la cuve. 
Cette cuve doit avoir un volume minimal calculé sur la base d'une dizaine de litres par alose.

Le dimensionnement de l'ascenseur (que ce soit pour le volume du bassin de stabulation et de la cuve ou pour la fréquence maximale de remontée de la cuve) doit prendre en compte les pics horaires de migration de l'alose qui peuvent atteindre $1.5 \%$ à $2 \%$ des passages totaux annuels.

L'espacement des barreaux des grilles de l'ascenseur doit être au maximum de $2.5 \mathrm{~cm}$.

La plus grande partie du débit d'attrait (et la totalité si les vitesses le permettent) doit être délivré en amont de la cage. La vitesse de l'écoulement à l'entrée du dispositif antiretour dans le bassin de stabulation doit être de l'ordre de $1 \mathrm{~m} / \mathrm{s}$.

La largeur minimale du canal assurant le transfert du poisson de l'ascenseur au plan d'eau amont doit être de $1.5 \mathrm{~m}$ et la vitesse de l'écoulement minimale de $0.30 \mathrm{~m} / \mathrm{s}$.

Les écluses "Borland" classiques sont à proscrire dans la mesure où leur efficacité est trop dépendante du comportement de l'espèce. On peut cependant envisager, dans le cas d'une chute réduite, et lorsque d'autres solutions paraissent difficilement envisageables, le recours à une écluse, à condition que son fonctionnement reste à surface libre (comme une écluse de navigation), en veillant à ce que le bassin de stabulation ait une longueur et un volume suffisants et en prenant soin de créer en phase de sortie un jet de surface suffisamment incitatif pour le poisson.

\section{ATTRAIT ET IMPLANTATION DES PASSES A ALOSES}

Par rapport aux passes à salmonidés, il convient d'être beaucoup plus exigeant sur l'implantation de la passe, sur la position des entrées et sur la qualité de l'attrait au pied de l'obstacle (en termes de vitesses, de débit, et de conditions hydrodynamiques).

De façon générale, les aloses prospectent en effet beaucoup moins que les salmonidés. Si pour le saumon on peut souvent se contenter d'une seule entrée, il n'en va pas de même pour l'alose. Dès que l'usine atteint une largeur d'une vingtaine de mètres, l'installation d'une galerie collectrice devient indispensable si l'on veut obtenir une efficacité suffisante. Dans le cas où il n'existe qu'une entrée unique, l'expérience montre que l'on est souvent obligé de "gérer" le fonctionnement des turbines pour faciliter l'accès de la passe aux aloses (cas des usines du Bazacle sur la Garonne et de Mauzac sur la Dordogne).

Les entrées principales doivent être situées le long des rives et les vitesses au niveau de ces entrées doivent rester importantes (de l'ordre de $2 \mathrm{~m} / \mathrm{s}$ ).

Il convient de mettre impérativement des grilles sur tout débit d'attrait complémentaire, ces grilles devant assurer non un blocage, mais un guidage du poisson vers l'échancrure ou la fente aval.

\section{EFFICACITÉ DES PASSES À ALOSES}

L'efficacité des passes pour l'alose est toujours moindre que pour les salmonidés. Si pour les salmonidés une efficacité de $90 \%$ à $100 \%$ est couramment obtenue, par contre, pour l'alose, une efficacité de $75 \%$ reste exceptionnelle, $50 \%$ est excellente et $10 \%$ à $20 \%$ fréquente.

II est cependant difficile d'évaluer l'efficacité d'une passe, même dans le cas le plus favorable où l'on dispose de plusieurs obstacles en série équipés de dispositifs de franchissement : il est rare en effet qu'il n'existe pas de zones favorables à la reproduction entre les différents ouvrages.

Sur les deux premiers obstacles (Essex et Pawtucket) situés sur la rivière Merrimack (côte Est des USA) et espacés d'une quinzaine de kilomètres, le pourcentage d'aloses passant d'un ouvrage au suivant est en moyenne de $10 \%$, il varie d'un minimum de $3 \%$ à un maximum de $23 \%$ (STOLTE, 1991).

Sur la Garonne, le pourcentage d'aloses ayant transité par l'ascenseur de Golfech et empruntant la passe du Bazacle (située à une centaine de km en amont) a varié entre 1989 et 1991 suivant les années de $17 \%$ à $32 \%$. Le pourcentage d'aloses ayant franchi l'une 
des passes du Bazacle et empruntant la passe du Ramier située immédiatement en amont a été évalué par échantillonnage à plus de $70 \%$ en 1990 (DARTIGUELONGUE, 1990). Ce pourcentage exceptionnel est lié à des conditions hydrologiques particulièrement favorables (débits très bas dans la Garonne) au cours desquelles les dispositifs de franchissement ont une attractivité optimale.

Sur la Dordogne, les passages d'aloses dans la passe à bassins de Mauzac varient de $0.5 \%$ à $30 \%$ de ceux observés dans l'ascenseur de Tuilières situé une quinzaine de kilomètres à l'aval. Cette variabilité importante s'explique en grande partie par l'hydraulicité de la Dordogne en période de migration (meilleurs passages par faibles débits) et par la perturbation plus ou moins importante selon les années apportée à l'attactivité de l'entrée de la passe de Mauzac par l'écoulement de la turbine Kaplan débouchant à proximité.

On entrevoit la difficulté de restauration de stocks importants d'aloses lorsqu'on est en présence d'obstacles en série sur un même cours d'eau et que toutes les zones de reproduction sont situées en amont.

\section{BIBLIOGRAPHIE}

BARIL D., 1988. Etude des conditions de franchissement par l'alose (Alosa ficta rhodannensis) d'une passe à ralentisseurs de fond expérimentale (site expérimental de Beaucaire). Rapp. final CSP, $35 \mathrm{p}$.

BEACH M.H., 1984. Fish pass design-criteria for the design and approval of fish passes and other structures to facilitate the passage of migratory fishes in rivers. Ministry of Agriculture, Fisheries and Food, Lowestoft, Fish. Res. Tech. Rep. 78, 45 p.

C.T.G.R.E.F., 1981. Migration des aloses dans le bas-Rhône : observations effectuées en 1979 et 1980. Rapp. non publié, $10 \mathrm{p}$.

DALLEY P.J., 1980. A review of fish passage facilities for american shad. Northeast Fish and Wildlife Conf., Ellenville, New-York, 32 p.

DARTIGUELONGUE J., 1990. Suivi de la migration de dévalaison et de montaison à la passe à poissons du Ramier au printemps 1990, $41 \mathrm{p}$.

DARTIGUELONGUE J., 1991. Contrôle du fonctionnement et de l'efficacité des passes à poissons installées au Bazacle en 1990, $31 \mathrm{p}$.

DARTIGUELONGUE J., 1992. Contrôle du fonctionnement et de l'efficacité des passes à poissons installées au Bazacle. Suivi de l'activité ichtyologique en 1991, 22 p.

DARTIGUELONGUE J., LARINIER M., TRAVADE F., 1992. Etude du comportement de l'alose dans la passe à poissons à l'usine de Tuilières sur la Dordogne. Rapp. CSPEDF, $55 \mathrm{p}$.

LITAUDON A., 1985. Observations préliminaires sur le franchissement du seuil de SaintLaurent-des-Eaux (Loire) par l'alose (Alosa alosa). Rapp. EDF HE/31/85-37, 63 p.

MONK B., WEAVER D., THOMPSON C., OSSIANDER F., 1989. Effects of flow and weir design on the passage behavior of american shad and salmonids in an experimental fish ladder. N. Am. J. Fish. Man., $9: 60-67$.

RIDEOUT S., THORPE L., CAMERON L., 1985. Passage of american shad in an Ice Harbor style fish ladder after flow modifications. Symp. on small hydropower and fisheries, Aurora, Colorado, 251-256.

SLATICK E., 1975. Laboratory evaluation of a Denil-type steeppass fishway with various entrance and exit conditions for passage of adult salmonids and american shad. MFR Pap. 1158, 17-26.

STOLTE L.W., 1990. Anodromous fish restoration program, Merrimack river. U.S. Fish and Wildlife Serv., Concord., New Hampshire, Annual Progress Rep., 19 p.

TRAVADE F., LAHINIEF M., TRIVELLATO D., DARTIGUELONGUE J., 1992. Conception d'un ascenseur à poissons adapté à l'alose (Alosa alosa) sur un grand cours d'eau : l'ascenseur à poissons de Golfech sur la Garonne. Hydroécol. Appl., 4 (1) : 91-119.

WEAVER C.R., 1965. Observations on the swimming ability of adult american shad (Alosa sapidissima). Trans. Am. Fish. Soc., 94 (4) : 382-385. 


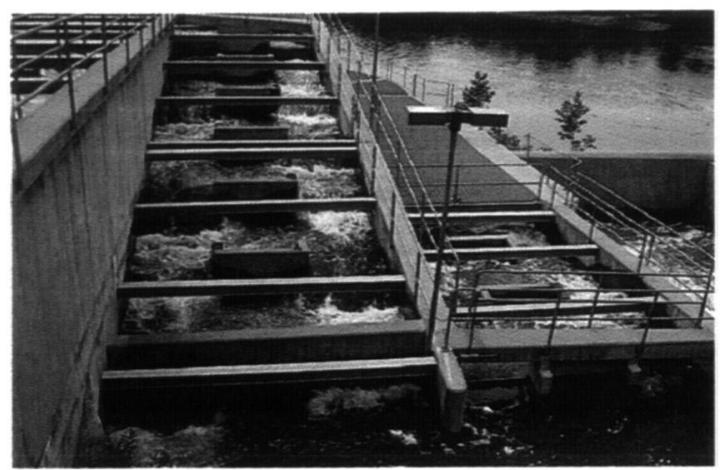

Photo 1 : Passe de Turners Falls sur la rivière Connecticut : une echancrure sur deux a été obturée et la charge augmentée pour faciliter le passage de l'alose.

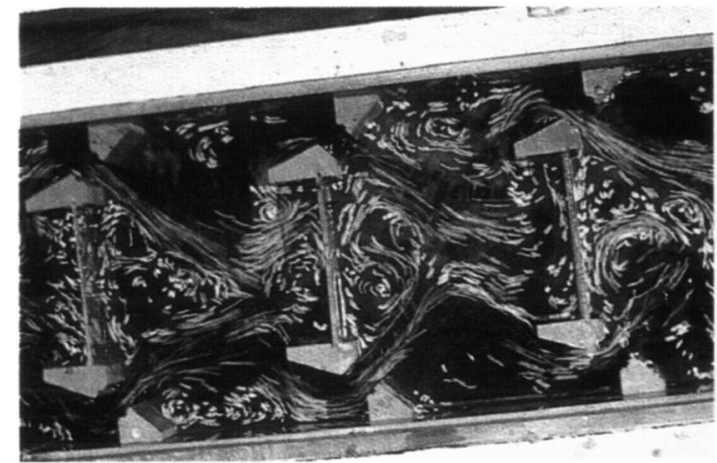

Photo 2 : Visualisation sur modèle réduit de l'écoulement dans la passe à deux fentes verticales de Bergerac sur la Dordogne.

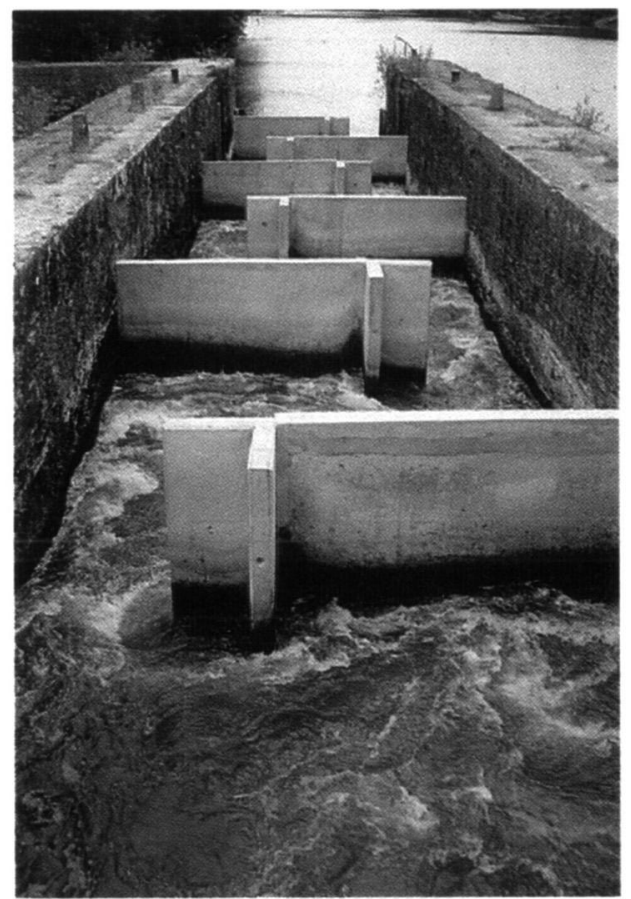

Photo 3 : Passe à échancrure latérale profonde sur le Tarn à SainteLivrade (Tarn). Cette passe à gros débit, installée dans une ancienne écluse de navigation, se révèle efficace pour l'alose. 


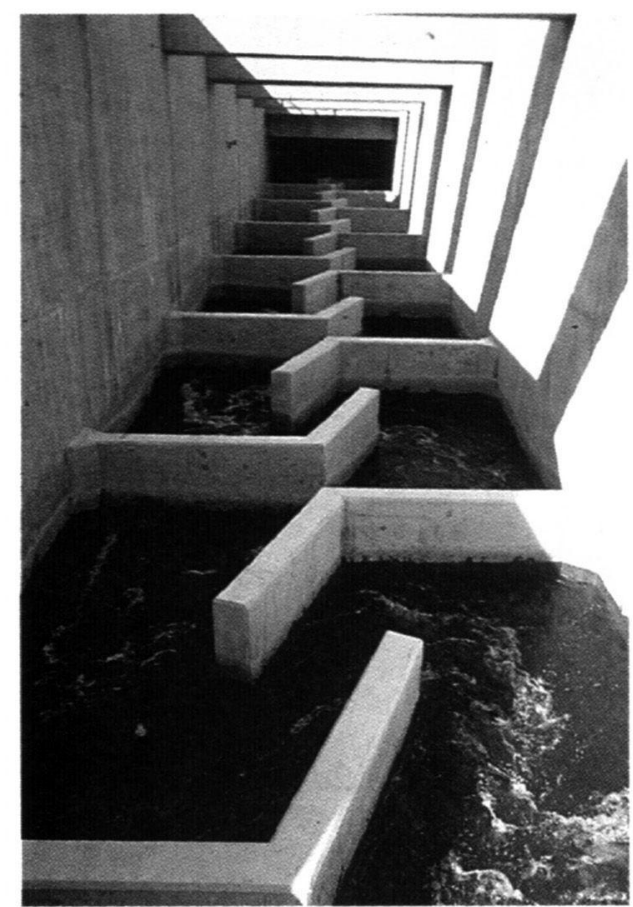

Photo 4 : Section de régulation à fentes verticales de la passe de Vernon sur la rivière Connecticut.

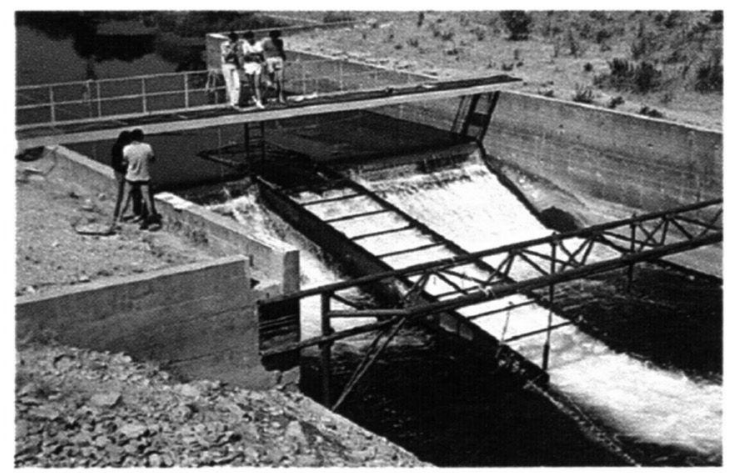

Photo 6 : Expérimentation d'une passe à ralentisseurs à chevrons épais sur un contre-canal du Rhône : on aperçoit à l'aval le filet guidant les aloses vers l'entrée de la passe.

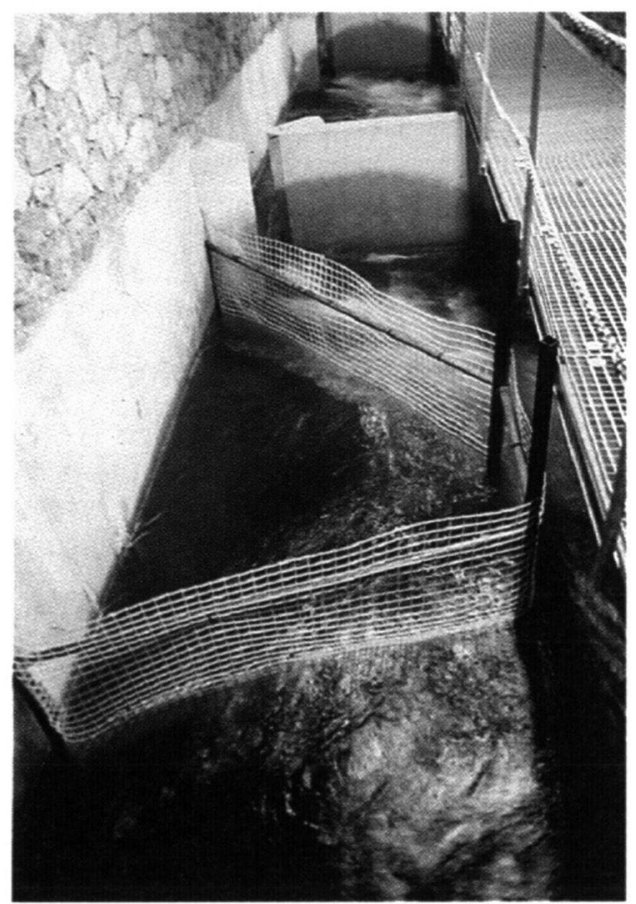

Photo 5 : Passe de Tuilières sur la Dordogne : un grillage interdit le piégeage des aloses dans les zones de recirculation et accélère leur passage en amont. 